\title{
Chronische Skrotalulcera
}

\section{Chronic Scrotal Ulceration}

Autoren

Institut

\section{Feldmann-Böddeker, T. Christoph}

Hautklinik (Chefärztin: Dr. med. I. Feldmann-Böddeker), DRK-Krankenhaus Chemnitz-Rabenstein

\section{Bibliografie}

DOI $10.1055 / \mathrm{s}-2007-966901$

Akt Dermatol 2007; 33:

368-370 @ Georg Thieme

Verlag KG Stuttgart · New York ISSN 0340-2541

Korrespondenzadresse

Dr. med. Ingrid FeldmannBöddeker

Hautklinik, DRK-Krankenhaus Chemnitz-Rabenstein

Unritzstraße 23

09117 Chemnitz

feldmann-böddeker.ingrid@

drk-chemnitz.de

\section{Zusammenfassung \\ $\nabla$}

Anhand der dargestellten Kasuistik wird insbesondere auf die differenzialdiagnostischen Überlegungen zur Abklärung von Skrotalulcera eingegangen.

\section{Einleitung \\ $\checkmark$}

Die Blickdiagnose chronische Skrotalulcera lässt zunächst an eine Vielzahl möglicher Differenzialdiagnosen denken. Neben zahlreichen Infektionskrankheiten müssen bei der Diagnostik auch Malignome und Autoimmundermatosen bedacht werden.

\section{Anamnese \\ $\nabla$}

Seit August 2006 bestehen zwei therapieresistente schmerzlose Ulcera am Skrotum des 56-jährigen Patienten. Über 6 Monate wurden Behandlungen mit unterschiedlichen antibiotischen und glukokortikoidhaltigen Externa durchgeführt. Seit 6 Wochen bemerkt der Patient eine Größenprogredienz der Ulcera, derzeit verwendet er lediglich eine Wundsalbe.

Im November 2006 wurde eine histologische Untersuchung einer Probebiopsie eines Skrotalulcus durchgeführt. Hier zeigte sich Ulcusrandgewebe mit Granulationsgewebe, fokalen kapillaritischen Zeichen, Plasmazellen und vereinzelten Eosinophilen. Kein Hinweis für Lymphom oder malignes Neoplasma.

Bekannt sind bei dem Patienten weiterhin eine diabetische Polyneuropathie bei Diabetes mellitus Typ 2, eine Hypercholesterinämie, eine arterielle Hypertonie, eine Prostatahyperplasie I- $\mathrm{II}^{\circ}$, eine Varikozele re. und eine Nebenhodenzyste li. Im Jahr 2005 erfolgte eine Behandlung wegen einer Borreliose.
Wegen der Therapieresistenz der Skrotalulcera erfolgte im Januar 2007 eine stationäre Einweisung in unsere Hautklinik.

\section{Hautbefund bei Aufnahme \\ $\nabla$}

Am linken Skrotum zwei scharf begrenzte, tiefreichende, nicht belegte Ulcera von $4 \times 3 \mathrm{~cm}$ und $2 \times 2 \mathrm{~cm}$ Durchmesser ( $\bullet$ Abb. 1 ), die umgebende Haut ist unauffällig, die Ulcera sind nicht schmerzhaft, Lymphknoten sind beidseitig inguinal nicht palpabel.

Das übrige Integument und die Mundschleimhaut weisen keine Hautveränderungen auf.

\section{Untersuchungsbefunde}

$\nabla$

Histologischer Befund einer Probebiopsie aus einem Skrotalulcus (Gemeinschaftspraxis für Pathologie und Zytologie, Dres. med. Beister/Neukirchner/Schweigert, Stollberg): Ulzerierende Dermatitis. Eine leukozytoklastische Vaskulitis liegt nicht vor. In der Defektzone imponieren fibrinoide Gefäßwandnekrosen und eine leukozytäre Infiltration, weiterhin ein perivasales, bis mäßig dichtes Rundzellinfiltrat. Differenzialdiagnostisch sollten ein Ulcus molle und ein Lymphogranuloma inguinale mit in die engere Wahl einbezogen werden. Gegen eine Lues spricht das Fehlen von plasmazellulären Infiltraten im entzündlichen Infiltrat. Kein Anhalt für Malignität.

Histologische Zweitbegutachtung der Probebiopsie des Skrotalulcus (Dermatologische Gemeinschaftspraxis Dres. Kutzner/Rütten, Mentzel et al., Friedrichshafen): Sekundäre Vaskulitis, die im Rahmen des chronisch-ulzerierenden Prozesses aufgetreten sein dürfte. Eine primär leukozytoklastische Vaskulitis liegt nicht vor. Mittels molekularpathologischer Methoden konnte keine Treponema pallidum-DNA, keine Herpes-sim- 


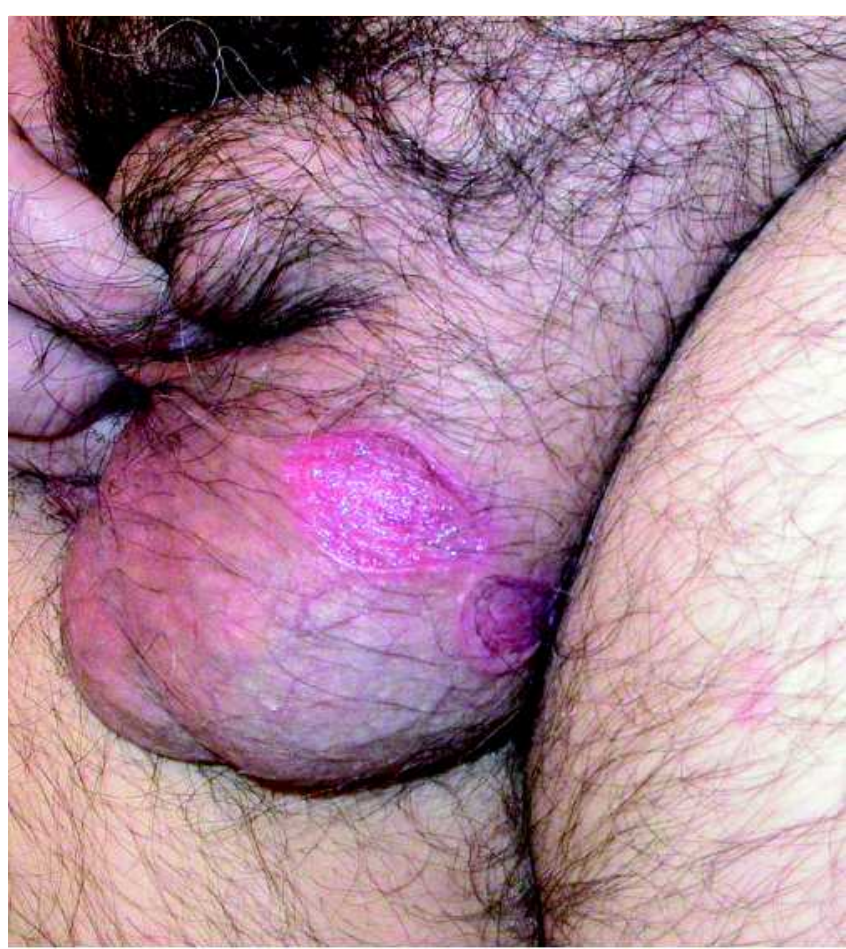

Abb. 1 Hautbefund bei Aufnahme: Chronische Skrotalulcera.

plex-Typ I- oder Typ II-DNA nachgewiesen werden. Es ergaben sich auch keine Hinweise für das Vorliegen einer Chlamydia trachomatis-, Haemophilus ducrey-, Pseudomonas-, Donovania granulomatis- oder Cytomegalovirus-DNA.

Immunfluoreszenzmikroskopische Untersuchung einer Probebiopsie eines Skrotalulcus: In den mit Anti-C 3-beschichteten Schnitten zeigte sich in der tiefen Epidermis eine Fluoreszenz entlang der Gefäßwände, ebenso an den mit Anti-Fibrinogen beschichteten Schnitten. Keine Fluoreszenz in den Schnitten, die mit Antikörpern gegen IgG und IgM beschichtet worden waren. Molekularbiologische Untersuchung einer erneuten Biopsie aus einem Skrotalulcus (Institut für Virologie/Medizinische Fakultät Carl Gustav Carus Dresden): Cytomegalie PCR negativ, EpsteinBarr-Virus PCR positiv.

\section{Labordiagnostik \\ $\nabla$}

Bakteriologische und mykologische Untersuchung von Abstrichen beider Skrotalulcera: Staphylococcus aureus und Enterococcus species, keine Anzucht von Anaerobiern oder Hefepilzen (Staphylococcus aureus war sensibel gegen Chloramphenicol und Cotrimoxazol, der andere Keim war sensibel gegen Amoxicillin, Ampicillin, Augmentan und Ciprofloxacin).

Lymphozytenstatus: Bei absolut normalen Lymphozyten liegen die Absolutwerte der Gesamt T-, B- und NK-Zellen im Normbereich. Die Helfer/Suppressor Ratio ist unauffällig mit 1,2.

Routinelabor: Differenzial-Blutbild $\mathrm{Hb} 8,4 \mathrm{mmol} / \mathrm{l}$, Erythrozyten 4,57 Tpt/l, sonst unauffällig. Unauffällig waren auch der Gerinnungsstatus, Albumin, Elektrolyte, Lebertransaminasen, alk. Phosphatase, Kreatinin, Harnsäure, GFR, CRP, TSH, T3, T4.

Glukose im Serum: 10,7 mmol/l, HbA1c 7,6\%.

Urinstatus: Glukose 5,55 mmol/l, ansonsten unauffällig. PSA: nicht erhöht.
Unauffällig waren auch ANA und Antikörper gegen dsDNS, weiterhin waren unauffällig die Serologie für HSV I, HSV II, Chlamydien IgG- und IgM-AK, HIV-AK, TPHA, Epstein-Barr-Virus-AK IgG und IgM, Borrelien-AK IgM, Borrelien-AK IgG 1:1280 (bekannte abgelaufene Borrelieninfektion).

Unauffällige Eiweißelektrophorese.

CMV-IgG 6,03 IE/ml (Referenzbereich <0,4 IE/ml), unauffällig waren CMV-KBR und CMV-IgM: Befund wie bei abgelaufener CMV-Infektion.

\section{Therapie und Verlauf \\ $\nabla$}

Entsprechend der bakteriologischen Abstrichuntersuchungen erfolgte zunächst eine Antibiose mit Ciprobay und Cotrimoxazol sowie eine wechselweise Lokaltherapie zunächst mit FucidineCreme, dann mit Triclosan 2\%-DAC-Basiscreme. Nachdem die Ulcera nach zweiwöchiger Behandlung in unveränderter Ausdehnung persistierten, erfolgte versuchsweise eine Therapie mit Protopic 0,1\% Salbe. Auch unter dieser Behandlung persistierte der Hautbefund.

Nach insgesamt vierwöchiger Behandlung traf der PCR-Befund ein, in dem DNA gegen Epstein-Barr-Virus nachgewiesen wurde. Nach einer jetzt fünftägigen Behandlung mit Aciclovir $250 \mathrm{mg} /$ $\mathrm{kg}$ Körpergewicht $3 \times$ tgl. als Kurzinfusion und weiterer Lokaltherapie mit Triclosan $2 \%$ in DAC-Basiscreme heilten die Ulcera innerhalb kurzer Zeit ab. Ein nochmalig durchgeführter HIV-Test war wiederum negativ.

\section{Diskussion}

$\nabla$

Die vorliegende Kasuistik soll auf die Vielzahl der zu diskutierenden und zu berücksichtigenden Differenzialdiagnosen bei chronischen und therapieresistenten Skrotalulcera hinweisen.

Ekthymata und Mykosen lassen sich durch entsprechende bakteriologische und mykologische Abstrichuntersuchungen klären. Mittels histologischer Untersuchung einer Probebiopsie sollten u. a. ein Karzinom, ein ulzeriertes Lymphom, eine Vaskulitis, ein Pyoderma gangraenosum, durch Histologie und Immunfluoreszenzhistologie weiterhin ein möglicherweise bestehender ulzerierender Lichen ruber erosivus oder Lupus erythematodes, ebenso ein bullöses Pemphigoid, ein Pemphigus vulgaris oder vegetans und ein extraintestinaler Morbus Crohn abgeklärt werden. Klinisch muss weiterhin an Artefakte und an einen Morbus Behcet gedacht werden. Auch die Abklärung einer Lues, eines Ulcus molle, eines durch Chlamydia trachomatis ausgelösten Lymphogranuloma inguinale sowie eines durch Calommatobacterium granulomatosis ausgelösten Donovania granulomatosis sollten ebenfalls diagnostisch abgeklärt werden. Schließlich muss bei persistierenden Ulcera im Genitoanalbereich auch an eine Herpes simplex-Virusinfektion durch HSV I oder HSV II, eine Zytomegalievirusinfektion oder wie im vorliegenden Fall diagnostiziert, an eine Epstein-Barr-Virus-Infektion gedacht werden. Die drei letztgenannten Infektionen treten in Zusammenhang mit Genitoanalulcera zumeist bei gleichzeitiger HIVInfektion auf. Sofern die Serologie wie auch in der vorliegenden Kasuistik für die zuletzt genannten Viren negativ ausfällt, empfiehlt sich auf jeden Fall auch der Versuch eines DNA-Nachweises, der möglicherweise vorhandenen Keime mittels PCR aus einer Probebiopsie aus der entsprechenden Läsion. 
Interessant ist bei der vorliegenden Diagnostik, dass die HIV-Serologie negativ war und sich auch kein Anhalt für einen Defekt im zellulären Immunsystem ergab. Da bei dem erwähnten Patienten möglicherweise noch keine Serokonversion erfolgt ist und er sich im sogenannten diagnostischen Fenster befindet, empfahlen wir auf jeden Fall weiter regelmäßige Kontrollen der HIV-Serologie durchzuführen.

\section{Abstract}

\section{Chronic Scrotal Ulceration}

$\nabla$

This case report is focused on the differential diagnosis of scrotal ulceration.

\section{Literatur}

1 Altmeyer $P$ et al. Dermatologische Differenzialdiagnosen. Heidelberg: Springer Medizin Verlag, 2007: 321 - 339

2 Schmoeckel C. Diagnostisches und Differenzialdiagnostisches Lexikon der Dermatologie. Stuttgart: Georg Thieme Verlag, 1997: 321 - 340

3 Braun-Falco O, Plewig G, Wolf $H$. Dermatologie und Venerologie, 3. Auflage. Heidelberg: Springer Medizin Verlag, 2005: 209- 254, $1036-1048$

4 Vartian CV, Septimus EJ. Genital ulceration in a young woman with infectious mononucleosis. Infections diseases in clinical practice. 1999; 8: $354-355$

5 Barnes $C J$, Alio AB et al. Epstein-Barr-Virus-associated genital ulcers: An Under-recognized disorder. Pediatric dermatology 2007; 24: $130-134$

6 Taylor S, Drake SM et al. Genital ulcers associated with acute EpsteinBarr virus infection. Sexually transmitted infections. 1998; 74: 296297

\section{Buchbesprechung}

Dermatopathologie

Kempf W, Hantschke M, Kutzner H, Burgdorf W

Darmstadt: Steinkopff, 2007. 296 S., 242 farbige Abb.

Geb. 99,95€

ISBN 978-3-7985-1647-2

Dermatologische Erkrankungen nehmen in ihrer Häufigkeit bedeutend zu, dadurch steigt auch die Anzahl von Hautgewebepräparaten, die histologisch beurteilt werden sollen.

Die Kenntnis der Dermatopathologie ist entscheidend für die Diagnostik der verschiedenen entzündlichen und nicht entzündlichen Dermatosen sowie malignen kutanen Erkrankungen. Besonders hervorzuheben ist im Gebiet der Dermatologie der enge Zusammenhang zwischen dem klinischen Bild und der histologischen Interpretation, bei dermatopathologischen Diagnosen ist die klinisch-pathologische Korrelation und damit die enge Zusammenarbeit zwischen dem Kliniker und Dermatopathologen von besonderer Bedeutung.

Das vorliegende deutschsprachige Werk verknüpft beide Bereiche, nämlich die einzelnen Krankheitsbilder auf der einen sowie die histologische Aufarbeitung auf der anderen Seite auf eine sehr anschauliche, übersichtliche Art und Weise. Von der Definition über die klinischen Erscheinungsbilder und Differenzialdiagnosen bis zur histopathologischen Beschreibung und Hinweise auf notwendige Zusatzuntersuchungen finden sich alle relevanten und häufigen Krankheitsbilder in einer gut strukturierten und übersichtlichen Form dargestellt. Den jeweiligen Krankheitsbildern gegenübergestellt finden sich Farbabbildungen in der Übersichtsvergrößerung sowie zusätzlich in Detailaufnahmen, alle mit genauen Erklärungen und entsprechenden Hinweispfeilen. Das Buch ist insgesamt in vier Gebiete gegliedert. Im ersten Abschnitt beschäftigen sich die Autoren mit dermatopathologischen Grundbegriffen, die Durchführung und Aufarbeitung von Hautbiopsien sowie mit histopathologischen Techniken. Der zweite Abschnitt ist den inflammatorischen und infektiösen Dermatosen gewidmet. Hierbei werden epidermale, dermale und subkutane Veränderungen erläutert. Der dritte Abschnitt beschreibt epitheliale Zysten und Pseudozysten. Im vierten Teil finden sich Informationen zu den häufigsten und wichtigsten Neoplasien sowie Hamartomen.

Zusätzlich findet sich am Anfang des Buches eine Tabelle mit den wichtigsten Spezialfärbungen und den entsprechenden dargestellten Strukturen sowie am Ende des Buches eine Auflistung verschiedener Krankheitsbilder, die mittels direkter Immunfluoreszenz ergänzend zur histologischen Aufarbeitung beurteilt werden können. Auch immunhistochemische Färbungen werden tabellarisch und übersichtlich dargestellt. Zusammenfassend findet der Leser ein übersichtlich gegliedertes Werk, welches die in der Praxis häufigsten dermatopathologischen Krankheitsbilder darstellt. Es bietet sowohl für den histopathologisch Ungeübten einen hervorragenden Zugang zu den Grundlagen der Dermatopathologie und ist zusätzlich für erfahrene Dermatopathologen ein wertvolles Nachschlagewerk.

C. Metzen, Wiesbaden 